\section{Análise da implantação do Programa de Controle da Tuberculose em unidades prisionais no Brasil}

\author{
Analysis of the implementation of the Tuberculosis \\ Control Program in Brazilian prisons
}

\author{
Análisis de la implantación del Programa de \\ Control de la Tuberculosis en cárceles en Brasil
}

Luisa Gonçalves Dutra de Oliveira 1

Sonia Natal 2,3

Luiz Antonio Bastos Camacho 2
${ }^{1}$ Instituto de Saúde Coletiva, Universidade Federal

Fluminense, Niterói, Brasil. 2 Escola Nacional de Saúde Pública Sergio Arouca, Fundação Oswaldo Cruz, Rio de Janeiro, Brasil.

${ }^{3}$ Departamento de Saúd Pública, Universidade Federal de Santa Catarina, Florianópolis, Brasil.

Correspondência L. G. D. Oliveira Rua Lopes Trovão 134, apto. 1702, Niterói, RJ 24220-071, Brasil.

luisa.dutra@gmail.com

\begin{abstract}
Tuberculosis control measures in Brazil's prison population have been regulated for ten years under the National Prison Health System Plan. Brazilian states have different organizational models for the Tuberculosis Control Program (TCP) in their prison systems. This study evaluated TCP implementation in prisons in two Brazilian states, using a multiple case study design with a qualitative approach and a log-frame analysis and assessment. According to predefined criteria, two state cases were selected, with two analytical units for each case and one prison hospital in Case 2. We identified partial program implementation in the Case 1 prisons and prison hospital and low implementation in non-hospital prison health services in Case 2. Lack of financial investment and resources, lack of integration between the courts and law enforcement system and health institutions, and poor access to health services in prisons were adverse factors for program implementation.
\end{abstract}

Turbeculosis; Prisons; Program Evaluation

\section{Resumo}

No Brasil, as ações de controle da tuberculose destinadas à população carcerária estão há dez anos regulamentadas pelo Plano Nacional de Saúde no Sistema Penitenciário. Os estados da federação têm modelos distintos de organização do Programa de Controle da Tuberculose (PCT) no sistema prisional. Este estudo avaliou o grau de implantação do PCT em unidades prisionais de dois estados brasileiros. Procedeu-se a um estudo de casos múltiplos com abordagem qualitativa e desenvolvimento de uma matriz de análise e julgamento. Segundo critérios pré-definidos foram selecionados dois casos estaduais, com duas unidades de análise para cada caso e um hospital penal no Caso 2. A implantação parcial do programa foi identificada nas unidades prisionais do Caso 1 e no hospital penal; um baixo nível de implantação foi constatado nas unidades prisionais não hospitalares do Caso 2. A falta de investimento financeiro e de recursos, a falta de integração entre as coordenações da justiça e da saúde e a dificuldade de acesso ao serviço de saúde foram alguns dos fatores desfavoráveis à implantação do programa.

Tuberculose; Prisões; Avaliação de Programas e Projetos de Saúde 


\section{Introdução}

A tuberculose (TB) foi considerada uma emergência global pela Organização Mundial da Saúde (OMS) em 1993. Os esforços empreendidos para a redução da carga da doença mostram alguns resultados positivos: as taxas de incidência e mortalidade têm apresentado queda global, e entre os 22 países que concentram $80 \%$ dos casos mundiais, sete já atingiram as metas de redução da incidência, prevalência e mortalidade previstas para 2015, entre eles o Brasil ${ }^{1}$. Um dos desafios para o controle da endemia é a elevada incidência entre populações de maior risco, entre elas a população prisional.

De acordo com a Organização Pan-Americana da Saúde (OPAS) 2, a incidência de TB nas unidades prisionais da América Latina é 22,2 vezes maior do que na população livre. No Brasil, estima-se que essa incidência seja 25 vezes maior 3 e que a prevalência de TB ativa varie de $2,5 \%$ a $8,6 \%$ entre os presos ${ }^{4}$. Sanchez 5 considera que a incidência pode estar subestimada, visto que a oferta e a qualidade do serviço de saúde no sistema prisional, a pouca motivação dos profissionais e a percepção dos presidiários quanto aos seus sintomas podem resultar na baixa detecção de casos.

$\mathrm{O}$ atraso no diagnóstico e no isolamento daqueles com a doença não detectada pode intensificar a transmissão a outros internos e aos funcionários das unidades prisionais, bem como aos contatos externos 6,7. O ambiente das prisões, em geral com celas superlotadas e ventilação inadequada, e os longos períodos de encarceramento também contribuem para a transmissão. A alta rotatividade dos prisioneiros entre as unidades prisionais pode resultar em falhas no tratamento, resistência aos fármacos e manutenção da transmissão ${ }^{6}$.

O Programa de Controle da Tuberculose (PCT) deve estar implantado no sistema prisional de forma a garantir rapidamente a detecção, o isolamento e o acompanhamento dos casos, bem como o planejamento da alta. A coordenação de esforços no âmbito penitenciário e extrapenitenciário é fundamental para o êxito do programa, assegurando ao indivíduo que foi libertado o acesso ao serviço de saúde e ao tratamento diretamente observado ${ }^{8}$.

No Brasil, foi estabelecido em 2003 o primeiro Plano Nacional de Saúde no Sistema Penitenciário (PNSSP). De acordo com esse plano, a gestão e gerência das ações e serviços de saúde nas penitenciárias são pactuadas entre as Secretarias Estaduais de Saúde (SES) e de Justiça e as secretarias municipais de saúde. O financiamento do programa é compartilhado pelos órgãos da justiça e da saúde nos níveis federal e estadual. A justiça/ administração penitenciária do estado provê os recursos físicos, humanos e materiais necessários às ações de saúde. No nível local, as secretarias municipais de saúde (SMS) dos municípios onde estão localizadas as prisões são responsáveis pelos exames complementares, as ações de maior complexidade e o atendimento hospitalar para os apenados. Às unidades de saúde das unidades prisionais cabe executar as ações de atenção primaria à saúde, ou seja, busca, diagnóstico, acompanhamento, tratamento e notificação dos casos diagnosticados, de acordo com a Portaria Interministerial no 1.777/2003 9. Esperam-se como efeitos a curto e médio prazos o aumento da detecção de casos, o diagnóstico e o tratamento precoces, e o aumento dos porcentuais de cura. A longo prazo, almeja-se a redução da incidência e da mortalidade por TB dentro dos presídios.

Este estudo teve como objetivo analisar a implantação do PCT no Sistema Penitenciário de dois estados brasileiros. Partiu-se do pressuposto de que a implantação das ações normatizadas pelo Programa Nacional de Controle da Tuberculose (PNCT) e pelo PNSSP atingiria os efeitos esperados.

A primeira etapa da avaliação, o estudo de avaliabilidade, constatou diferentes modelos de organização do PCT no sistema prisional dos dois casos selecionados 10 .

\section{Métodos}

Um estudo de casos múltiplos 11 foi desenvolvido no período de dezembro de 2010 a junho de 2012 . com abordagem qualitativa, para operacionalizar o modelo lógico e o modelo teórico do programa, apresentados no estudo de avaliabilidade 10 . Os casos selecionados foram o Sistema de Saúde Penitenciária de dois estados brasileiros com grande população carcerária, tendo como critério de seleção altas taxas de incidência de TB nas prisões. As unidades de análise foram duas unidades prisionais para cada caso e um hospital penal em um dos casos. As unidades prisionais, selecionadas conjuntamente com os coordenadores de saúde penitenciária, tinham o PCT implantado, a possibilidade de realizar o diagnóstico laboratorial e um grande número de casos novos de TB registrados. Foram consideradas somente unidades com detentos do sexo masculino, que compõem a grande maioria da população encarcerada, e por ser o gênero com maior prevalência de TB. A análise dos dois casos foi estruturada para identificar os aspectos positivos do controle da TB e as oportunidades de melhora nas unidades prisionais. 
Os dados primários foram obtidos por meio de entrevistas realizadas com sete gestores e diretores, 37 profissionais de saúde do sistema penitenciário, 14 agentes de segurança e 42 detentos em tratamento da TB, e também mediante a observação sistemática da execução das ações do programa realizada em 19 visitas às unidades prisionais selecionadas. Os dados de fontes secundárias foram coletados dos documentos $\mathrm{e}$ normas técnicas do programa, legislação relativa à saúde penitenciária, relatórios, livros de registro, prontuários e do Sistema de Informação de Agravos de Notificação (SINAN) nacional.

Para determinar o grau de implantação do PCT nas unidades de análise selecionadas, utilizou-se a matriz de análise e julgamento (Tabela 1), com cinco categorias: acesso, infraestrutura, busca de casos, diagnóstico e acompanhamento, tratamento e assistência farmacêutica. Tais categorias foram validadas anteriormente em estudos avaliativos do PCT para a população em geral 12,13. Na categoria acesso foram consideradas a disponibilidade de recursos e sua relação com a necessidade da clientela; a acessibilidade, que abrange a localização dos serviços e a necessidade de transporte; e a acomodação, ou seja, o modo como os recursos são organizados para atender aos usuários 14. A infraestrutura necessária para as ações de atenção básica previstas envolveu a quantidade e qualidade dos recursos humanos e materiais, bem como a utilização do incentivo financeiro previsto na legislação para as unidades de saúde das unidades prisionais. A busca de casos consistiu nas ações destinadas à detecção precoce: abordagem sistemática dos detentos, ações de educação e informação, exame de ingressos e investigação de contatos. Na categoria diagnóstico e acompanhamento foram considerados os exames necessários para a confirmação e o seguimento dos casos, além da notificação dos mesmos. A categoria tratamento e assistência farmacêutica enfocou a forma de administração da terapêutica, o fornecimento dos fármacos, os registros da evolução do tratamento e as ações de comunicação necessárias à continuidade do tratamento nos casos de transferência ou libertação.

Para cada categoria foram elaborados indicadores e estabelecida para cada um deles uma pontuação máxima. Cada categoria recebeu a mesma pontuação estimada e a variação ocorreu internamente na distribuição dos valores por indicador. Para estimar a carga valorativa dos indicadores foram consideradas como padrão as metas preconizadas pelo Ministério da Saúde e Ministério da Justiça, além das considerações dos envolvidos na implantação do programa.

A proporção do somatório dos pontos observados nas categorias em relação à pontuação es-
Tabela 1

Pontos de corte para a classificação dos casos conforme os graus de implantação.

\begin{tabular}{lc}
\hline Pontos de corte & Grau de implantação \\
\hline $76 \%$ a $100 \%$ & Implantado \\
$51 \%$ a $75 \%$ & Parcialmente Implantado \\
$26 \%$ a $50 \%$ & Baixo nível de implantação \\
Abaixo de $26 \%$ & Implantação incipiente \\
\hline
\end{tabular}

timada, por cada unidade de análise, determinou o grau de implantação: GI = $\left(\sum\right.$ ponto observa$\mathrm{d} \delta \sum$ ponto estimado) $\mathrm{x} 100$. As proporções foram estratificadas em quartis para a classificação do GI, como apresentado na Tabela 1. Os pontos de corte baseados nos quartis, já empregados em estudos de avaliação anteriores, permitiram uma discriminação satisfatória do GI dos casos.

A análise simples dos casos, feita de forma descritiva com triangulação de dados primários e secundários, utilizou a análise de conteúdo de Bardin 15 para os dados qualitativos. A análise sistemática e objetiva das informações obtidas, com base nas categorias determinadas no estudo, permitiu identificar elementos para descrever as ações de controle da TB desenvolvidas. Os extratos das entrevistas transcritas e dos registros das observações de campo que expressavam as opiniões e as ações dos atores do programa e os trechos retirados dos documentos analisados foram organizados de forma manual, cada texto isoladamente 16 . A análise por categorias funcionou por operações de desmembramento do texto e reagrupamento por unidades de registro 15 . Na análise imbricada dos casos, o exame minucioso das categorias de análise permitiu verificar os padrões encontrados e a existência de coincidências e divergências entre eles.

Para fortalecer a validade interna do estudo, utilizou-se a triangulação dos dados de maneira a verificar de forma contínua a confiabilidade e a interpretação das informações coletadas 16. A adequação entre o modo de análise e o modelo teórico proposto no estudo 11,17 também contribuiu na validade interna. A validade externa no estudo de casos múltiplos se verifica pela possibilidade de replicação, em outras unidades de análise, do modelo de avaliação 11 .

Este trabalho foi aprovado pelo Comitê de Ética em Pesquisa da Escola Nacional de Saúde Pública Sergio Arouca, Fundação Oswaldo Cruz (parecer no 13.477/12). As Secretarias Estaduais de Administração Penitenciária dos dois estados aprovaram formalmente a realização da pesquisa. Considerando que a população encarcerada 
pode estar suscetível a pressões, foi assegurada a obtenção para cada detento de consentimento livre e esclarecido para participar do estudo e a garantia da confidencialidade dos dados e sua utilização exclusivamente para fins de pesquisa.

\section{Resultados}

\section{Grau de implantação}

A Tabela 2 apresenta a matriz de análise e julgamento com a pontuação observada em cada categoria. Os resultados do grau de implantação mostraram que o PCT estava "parcialmente implantado" nas unidades de análise 1 e 2 (UA1 e UA2) do Caso 1, com proporções de 69\% e 63\%, respectivamente. No Caso 2, o hospital penal (UA3) atingiu $71 \%$, sendo o programa nesta UA tido como "parcialmente implantado". As UA4 e UA5 do Caso 2 apresentaram "baixo nível de implantação", com 48\% e 39\%, respectivamente.

No Caso 1, o estado tem um grande número de unidades prisionais dispersas por todo o território, em municípios com população que variava de 28 mil a mais de 11 milhões de habitantes. As unidades prisionais do Caso 1 selecionadas para este estudo estavam localizadas: uma na região metropolitana e a outra no interior do estado. No Caso 2, o estado tem a maioria das unidades prisionais localizadas em quatro municípios da região metropolitana, com uma população total de pouco mais de 7 milhões de habitantes. As unidades prisionais selecionadas para este estudo ficavam na capital do estado (duas) e na região metropolitana (uma). Todas as UA selecionadas foram prisões masculinas de regime fechado e/ou semiaberto, superlotadas (com exceção do hospital) e com um número significativo de detentos em tratamento da TB (Tabela 3). A população carcerária em ambos os Casos era composta predominantemente por jovens, com baixa escolaridade, provenientes de locais com más condições de vida e com maior incidência de TB. Dados do Departamento Penitenciário Nacional mostram que $54,8 \%$ dos presos no Brasil tinham, em dezembro de 2012, entre 18 e 29 anos de idade, $63 \%$ não tinham completado o Ensino Fundamental e 54,5\% eram provenientes das regiões metropolitanas do país.

Nas unidades prisionais estudadas predominou o regime fechado. Mesmo os detentos com direito ao regime semiaberto ou aberto tinham seu benefício postergado devido à morosidade jurídica. A permanência desses presos nas celas favorece a superlotação e, consequentemente, a maior possibilidade de transmissão da TB.

Na Tabela 4 são apresentados os aspectos favoráveis e os desfavoráveis à implantação das ações de controle da TB nas unidades prisionais, semelhantes nos dois casos selecionados.

Os aspectos divergentes entre o Casos 1 e 2 são apresentados na Tabela 5. No Caso 1, destacam-se aspectos mais favoráveis para o diagnóstico e tratamento. No Caso 2, a utilização de radiografia destaca-se nos aspectos favoráveis; entre os aspectos desfavoráveis está a ausência de busca ativa periódica, entre os ingressos e entre os contatos dos casos diagnosticados.

Matriz de análise e julgamento: grau de implantação do Programa de Controle da Tuberculose (PCT) nas unidades prisionais.

\begin{tabular}{|c|c|c|c|c|c|c|c|}
\hline \multirow[t]{4}{*}{ Categorias/Dimensão implantação } & Fontes e tipos & PE (\%) & \multicolumn{5}{|c|}{$\mathrm{PO}(\%)$ * } \\
\hline & \multirow[t]{3}{*}{ de coleta } & & Caso & Caso & Caso & Caso & Caso \\
\hline & & & 1 & 1 & 2 & 2 & 2 \\
\hline & & & UA1 & UA2 & UA3 hospital & UA4 & UA5 \\
\hline Acesso & Entrevistas com: gestores, & $10(100)$ & 50 & 60 & 80 & 55 & 25 \\
\hline Infraestrutura & profissionais de saúde, agentes & $10(100)$ & 45 & 25 & 75 & 45 & 50 \\
\hline Busca de casos & penitenciários e sentenciados. & $10(100)$ & 85 & 65 & 50 & 40 & 30 \\
\hline Diagnóstico e acompanhamento clínico, & Observação direta. & $10(100)$ & 75 & 75 & 70 & 70 & 60 \\
\hline laboratorial e radiológico & Análise de prontuários.Análise & & & & & & \\
\hline \multirow[t]{2}{*}{ Tratamento e assistência farmacêutica } & documental. & $10(100)$ & 90 & 90 & 80 & 30 & 30 \\
\hline & Livro de registro. & & & & & & \\
\hline Gl ( $\sum$ Observado $/ \sum$ Estimado $) \times 100$ & & $50(100)$ & $69 \%$ & $63 \%$ & $71 \%$ & $48 \%$ & $39 \%$ \\
\hline
\end{tabular}

GI: grau de implantação; PE: pontuação estimada; PO: pontuação observada; UA: unidades de análise.

$\star P O=\left(\sum P O / \Sigma P E\right) \times 100$. 
Tabela 3

Características das unidades de análise (UA).

\begin{tabular}{|c|c|c|c|c|c|}
\hline \multirow[t]{2}{*}{ Características das UA } & \multicolumn{2}{|c|}{ Caso 1} & \multicolumn{3}{|c|}{ Caso 2} \\
\hline & UA1 & UA2 & UA3 & UA4 & UA5 \\
\hline Regime & $\begin{array}{l}\text { Fechado e } \\
\text { semiaberto }\end{array}$ & Fechado & Fechado & $\begin{array}{c}\text { Semiaberto e } \\
\text { aberto }\end{array}$ & Fechado \\
\hline Capacidade & 852 & 1.200 & 105 & 433 & 1.400 \\
\hline Lotação * & 1.200 & 2.200 & 105 & 497 & 1.850 \\
\hline Capacidade das celas & 3 a 6 & 6 & Em torno de 10 & 18 a 52 & 50 \\
\hline Lotação por cela * & 10 a 12 & 12 a 15 & 10 & 15 a 99 & 70 a 100 \\
\hline Casos de TB ** & 10 & 20 & 105 & 4 & 12 \\
\hline
\end{tabular}

* Lotação aproximada durante a coleta de dados na unidade prisional;

** Número de casos no período de coleta de dados.

Tabela 4

Aspectos semelhantes dos Casos 1 e 2 .

\begin{tabular}{|c|c|}
\hline Favoráveis & Desfavoráveis \\
\hline \multirow[t]{3}{*}{$\begin{array}{l}\text { Exame baciloscópico para diagnóstico e } \\
\text { acompanhamento }\end{array}$} & $\begin{array}{l}\text { Acesso à unidade de saúde decidido por agentes de segurança e/ou } \\
\text { presidiários }\end{array}$ \\
\hline & Carência de transporte e escolta dificultam o sistema de referência \\
\hline & Ventilação inadequada na unidade de saúde e na unidade prisional \\
\hline \multirow[t]{3}{*}{ Não houve falta de medicamentos para TB } & Dificuldade para execução do incentivo financeiro \\
\hline & Falta de recursos para as ações de saúde no sistema penitenciário \\
\hline & Equipe de saúde desmotivada \\
\hline \multirow[t]{4}{*}{ Notificação dos casos } & Equipe de saúde incompleta em uma das unidades de análise, com \\
\hline & número insuficiente de médicos e de profissionais de enfermagem \\
\hline & Dificuldade de comunicação nos casos de libertação ou transferência \\
\hline & Condições de encarceramento (regime fechado + superlotação) \\
\hline
\end{tabular}

\section{Ações de implantação do programa observadas}

O acesso dos internos às unidades de saúde das unidades prisionais dos Casos 1 e 2, por demanda espontânea, se dava pela solicitação feita ao agente de segurança, do envio de bilhetes ou da inclusão do nome em uma lista preparada pelos próprios presidiários.

O tempo de espera relatado para o atendimento foi de uma semana, na maior parte das vezes, podendo variar de acordo com a gravidade do caso e dos sintomas apresentados, visto que a decisão sobre quem seria encaminhado à unidade de saúde competia, por vezes, ao grupo de presos. Na UA5, Caso 2, o tempo de espera para encaminhamento à unidade de saúde podia prolongar-se por até quatro meses, em virtude da grande demanda e do número reduzido de profissionais de saúde.

Em geral, o atendimento inicial era feito por um profissional de enfermagem que podia decidir pelo encaminhamento à consulta médica, se julgasse necessário. A presença do médico na unidade de saúde variou entre as unidades prisionais de uma a cinco vezes por semana.

O deslocamento dos internos das celas para as unidades de saúde das unidades prisionais oferecia menos obstáculos no Caso 1 , já que estas unidades estavam no mesmo pavilhão das celas e não havia necessidade de escolta. Já no Caso 2, as unidades ficavam em local externo ou em outro pavilhão, tornando necessária a escolta dos presos. A falta de meio de transporte e de escolta foi, em ambos os casos, empecilho frequente para a realização de exames especializados na rede 
Aspectos divergentes entre os Casos 1 e 2

\begin{tabular}{ll}
\hline Caso 1 & Caso 2 \\
\hline Favoráveis & \\
\hline Localização da unidade de saúde no mesmo pavilhão dos & Unidades de saúde em boas condições \\
alojamentos & Radiografia para todos os casos \\
Publicação dos casos em tratamento (acesso diário) & Rastreamento radiológico \\
Campanhas de busca ativa & Acompanhamento dos casos por equipe específica \\
Exame dos ingressos & \\
Exame dos contatos & \\
Oferta de teste anti-HIV para todos & \\
Cultura e TSA para todos os casos diagnosticados e & \\
sintomáticos & \\
Tratamento diretamente observado & \\
Acompanhamento dos casos pela equipe de saúde da & \\
unidade prisional &
\end{tabular}

Desfavoráveis

Unidades de saúde em precárias condições

Radiografia extramuros para poucos casos
Necessidade de escolta para acessar a unidade de saúde

Carência de transporte e escolta dificultam o diagnóstico

e o início do tratamento para tuberculose

Detecção passiva dos casos

Rastreamento radiológico interrompido

Falta de recursos para oferta de teste anti-HIV, cultura e

TSA para todos os casos

Tratamento autoadministrado

Dispensação semanal dos medicamentos

Equipe de saúde da unidade prisional como

"coadjuvante" no tratamento para tuberculose

TSA: teste de sensibilidade aos antimicrobianos.

de saúde extramuros. No Caso 2, esse empecilho também dificultava o exame do sintomático respiratório e o diagnóstico da $\mathrm{TB}$, uma vez que atrasava a ida do detento ao hospital penal.

Em todas as unidades prisionais estudadas, o acompanhamento rotineiro do tratamento era feito por profissionais de enfermagem. A consulta médica estava prevista apenas para o início do tratamento e para o momento da alta. Na UA2 do Caso 1, entretanto, a consulta médica era mensal, feita com a participação do enfermeiro. No hospital penal, Caso 2, o acompanhamento médico do paciente era feito semanalmente. Para os pacientes não hospitalizados do Caso 2, o acompanhamento periódico dos pacientes nas unidades prisionais da região metropolitana era feito pela equipe DOTS (directly observed treatment, short- course), como era chamada a equipe vinculada à Coordenação de Saúde Penitenciária, composta por uma enfermeira, uma psicóloga e seis auxiliares de enfermagem que se dedicavam desde 2010 às ações de controle da TB.
A estrutura física das unidades de saúde era precária: consultórios sem ventilação adequada, sem local para a coleta de escarro e carência de recursos materiais básicos como mesa para exame e negatoscópio. As unidades de saúde do Caso 1 estavam em mau estado de conservação.

A equipe de saúde era insuficiente em duas das unidades prisionais estudadas, a UA2 do Caso 1 e UA5 do Caso 2. No hospital penal, mais da metade dos profissionais de saúde receberam algum tipo de treinamento sobre TB nos últimos dois anos. No restante das unidades prisionais, a maior parte não havia recebido qualquer tipo de capacitação e não utilizava o manual de recomendações para o controle da TB. A desmotivação dos profissionais em virtude dos baixos salários e das precárias condições de trabalho foi relatada em todas as unidades estudadas.

A busca ativa sistemática era realizada em forma de campanhas nas UA do Caso 1, com a divulgação dos sintomas da TB e oferta de exame baciloscópico e cultura para todos os sintomáti- 
cos. No Caso 2, não havia busca ativa nas unidades estudadas, a identificação de casos se dava a partir da demanda espontânea.

O exame do preso que ingressava no sistema prisional era feito em até sete dias nas UA do Caso 1, pelo médico ou enfermeiro. Procediase à investigação dos sintomas respiratórios e da história prévia de TB. Aos sintomáticos eram solicitados o exame baciloscópico, a cultura e o teste de sensibilidade aos antimicrobianos (TSA) e ofertado o teste anti-HIV. No Caso 2, a busca de casos entre os ingressos com exame físico, baciloscopia e raio-X de tórax foi possível no período de agosto de 2010 a fevereiro de 2011, em que a entrada dos presos era centralizada. Com o estabelecimento de outra entrada para os ingressos, houve dificuldade para operacionalizar os exames devido a questões logísticas e à alta rotatividade na unidade.

O exame dos contatos dos casos diagnosticados era feito nas duas unidades estudadas do Caso 1, mas não acontecia no Caso 2, em virtude do grande número de detentos encerrados em uma mesma cela, o que dificultava ou inviabilizava a ação. Mais da metade dos pacientes em tratamento entrevistados na UA2 do Caso 1 disseram ter sido convocados a realizar o exame no momento da admissão ou por serem contatos de casos na mesma cela ou galeria, e se consideravam assintomáticos na ocasião.

Os contatos externos, familiares e visitantes, não são abordados diretamente pelos profissionais de saúde do sistema prisional. Na UA3, Caso 2, 62,5\% dos pacientes entrevistados disseram ter recebido informações para orientar seus familiares a procurarem as unidades de saúde externas para exame. Já nas UA4 e 5, 25\% e 36,4\% dos pacientes entrevistados tinham essa informação, respectivamente. No Caso 1, a situação é semelhante: enquanto na UA1 75\% dos internos orientavam seus contatos externos, na UA2 esta proporção foi de $45,5 \%$. De maneira geral, os internos solicitavam que houvesse mais atividades de informação e divulgação. Atividades desse tipo só foram descritas na UA5, feitas periodicamente pela enfermeira da equipe DOTS, com o objetivo de aumentar a adesão ao tratamento e a detecção precoce dos casos.

O exame baciloscópico para diagnóstico e acompanhamento mensal, bem como a cultura e o TSA, eram feitos para todos os pacientes nas UA do Caso 1. Os laboratórios municipais e estaduais estabeleciam um número máximo de exames semanais para cada unidade prisional. A cota de baciloscopias era, no entanto, suficiente e não havia dificuldade em incluir mais amostras, caso necessário. A cultura e o TSA também eram garantidos para todos, ainda que houvesse também cotas, o que, por vezes, gerava atraso. Já no Caso 2, a baciloscopia diagnóstica e mensal estava assegurada, mas a cultura e o TSA não eram realizados para todos, priorizando-se os casos de retratamento ou persistência de positividade na baciloscopia após dois meses de tratamento. A carência de recursos materiais no Caso 2 também impossibilitava a oferta do exame anti-HIV para todos, o que não era dificuldade no Caso 1. O exame radiológico, por outro lado, era feito para todos os sintomáticos respiratórios do Caso 2 que compareciam ao hospital penal para avaliação médica. No Caso 1, 26,3\% dos detentos em tratamento relataram ter sido submetidos à radiografia para diagnóstico.

A centralização dos exames no hospital penal e o acompanhamento dos casos por equipe externa no Caso 1 resultavam no desconhecimento, por parte da equipe de saúde da própria unidade prisional, da situação dos pacientes. Os profissionais de saúde consideravam que os pacientes com $\mathrm{TB}$, embora recebessem o tratamento na unidade prisional, estavam sob responsabilidade do hospital penal, considerando-se apenas coadjuvantes no acompanhamento dos casos.

Nos estados estudados, a totalidade das unidades prisionais apresentava cobertura de baciloscopia para os casos novos, em 2011, de 97,9\% no Caso 1 e de 90,4\% no Caso 2. Os percentuais de testagem para HIV e cultura tinham diferenças significativas: $82,1 \%$ e $36,3 \%$ de exames anti-HIV solicitados nos Casos 1 e 2, respectivamente, e $81,9 \%$ e $13,7 \%$ de culturas realizadas nos Casos 1 e 2, respectivamente, no mesmo ano de 2011 (Área Técnica de Saúde no Sistema Penitenciário, Ministério da Saúde. Dados sobre TB prisional; 2013).

A notificação de todos os casos diagnosticados era feita ao município pelas unidades de saúde das unidades prisionais do Caso 1, e pelo hospital penal do Caso 2.

O tratamento diretamente observado por profissional de saúde era realizado nas UA do Caso 1 nos dias úteis. Nos fins de semana, a entrega do medicamento ficava sob a responsabilidade do agente de segurança. No Caso 2, a dispensação dos medicamentos era feita semanalmente por profissional de saúde, sendo o tratamento autoadministrado. Considerando o conjunto de todas as unidades prisionais dos dois estados, a proporção dos casos de TB que recebiam tratamento diretamente observado foi, em 2011, de $56,1 \%$ no Caso 1, e de 14,4\% no Caso 2 (Área Técnica de Saúde no Sistema Penitenciário, Ministério da Saúde. Dados sobre TB prisional; 2013).

Quando da transferência de detentos para outra unidade prisional do Caso 1, acompanhava-os um prontuário que continha também a 
ficha de notificação, a receita, os resultados de exames e ficha de controle do tratamento diretamente observado, bem como um suprimento de medicamentos suficiente para 15 dias. Quando ocorria a libertação de um presidiário em tratamento, este recebia uma carta de encaminhamento à unidade de saúde externa e medicamentos em quantidade suficiente para 15 dias. Além disso, a unidade prisional comunicava a libertação do caso de TB à vigilância epidemiológica do município. Já no Caso 2, a comunicação entre as unidades de saúde das UP não era feita rotineiramente, em virtude da carência de recursos humanos e materiais. Com o objetivo de garantir o fluxo de informações nas transferências internas e externas, era fornecido ao preso, desde o início do tratamento, um registro com informações sobre seu diagnóstico, tratamento e resultados de exames. Quando tomavam ciência das transferências, os profissionais da equipe DOTS procuravam os pacientes nas unidades prisionais de destino para assegurar a continuidade do tratamento. Não havia qualquer rotina de comunicação entre o sistema penitenciário e o município ou o estado, que possibilitasse comunicar as altas.

\section{Discussão}

Este trabalho mostrou que as ações do PCT não estão totalmente implantadas nas prisões estudadas em dois dos estados com maior população prisional, altas taxas de incidência de tuberculose e situação econômica melhor do que a média do país. Reichard et al. 18 também encontraram variações e deficiências na implantação das normas de controle da TB nas prisões americanas, como: demora no rastreamento e isolamento dos casos suspeitos e dificuldade de acompanhamento para os casos transferidos entre unidades prisionais ou libertados.

O hospital penal para tratamento dos casos de TB, unidade com o maior grau de implantação neste estudo, dispunha de mais recursos humanos, laboratoriais e radiológicos, e oferecia acesso mais fácil dos pacientes internados aos profissionais de saúde. Estudos realizados em outros países mostram que o tratamento da TB na população carcerária é feito, na maioria das vezes, em unidades ambulatoriais 19,20,21. Um artigo publicado em 2004 descreve que o tratamento nas prisões do Malawi era feito, nas primeiras duas semanas, em hospitais próximos às unidades prisionais 22 . Um estudo posterior feito no mesmo país ${ }^{23}$, revelou que o tratamento da TB era realizado nas próprias prisões e que poderia haver a transferência de um prisioneiro com o diagnóstico de TB pulmonar bacilífera para a prisão mais próxima que dispusesse de uma equipe de saúde.

São aspectos convergentes entre os dois casos estudados o acesso inicial do preso à unidade de saúde dependendo da autorização do agente de segurança e/ou de um grupo de detentos; o número de profissionais de saúde desmotivados com as condições de trabalho e sem treinamento periódico; o investimento insuficiente; e a carência de transporte e escolta adequados às ações de saúde extramuros. Souza et al. ${ }^{24}$, em um estudo realizado em prisões do Nordeste do Brasil, verificaram que a decisão de permitir o acesso aos serviços de saúde era tomada por profissionais da área de vigilância e disciplina, e que este era um fator que contribuía para o atraso no diagnóstico e para a dificuldade de controle da TB no sistema prisional. A insuficiência de recursos humanos e materiais mostrou-se, em outro estudo, prejudicial à execução das ações de saúde 25 . A falta de coordenação entre as unidades de saúde das prisões e o sistema de saúde extramuros resultou, em estudo apresentado por Castro 26, na indefinição de responsabilidades nas ações de saúde nas prisões, comprometendo também, de acordo com outros estudos, o sistema de informação e a continuidade do tratamento dos egressos 18,20,24. O sistema de registro e notificação está presente, mas ainda carece de aperfeiçoamento.

O exame de ingressos, a busca ativa dos sintomáticos e o tratamento diretamente observado eram realizados de forma sistemática nas UA do Caso 1. Entretanto, a entrega dos medicamentos pelo agente de segurança, como acontecia nos fins de semana nessas unidades, não é recomendada pelo PNCT 3, por descaracterizar o tratamento diretamente observado como uma ação exclusiva dos profissionais de saúde e pelo risco de constituir um elemento de pressão. Alguns estudos destacaram que manter a continuidade das ações de busca ativa e de supervisão do tratamento é importante para aumentar a detecção de casos e as taxas de cura e para reduzir o abandono 22,23. Entretanto, outros trabalhos recentes 27,28,29,30 consideram que não há evidências significativas de melhores resultados de tratamento entre os casos detectados por meio de busca ativa. Segundo Golub \& Dowdy 28, ainda que o rastreamento possa reduzir o atraso no diagnóstico, evitar o agravamento da doença e reduzir a transmissão, principalmente em populações sob maior risco de infecção e adoecimento, a falta de recursos e as dificuldades operacionais podem prejudicar a efetividade dessa estratégia. Lönnroth et al. 29 ressaltam que os exames para confirmação diagnóstica devem ser garantidos aos suspeitos de TB ativa identificados pela triagem. 
A baciloscopia, feita nas UA do Caso 1 em ingressos, contatos e detentos com algum sintoma, mostrou-se um exame com pouca sensibilidade para o rastreamento e o diagnóstico. Como a investigação bacteriológica incluía também a cultura, aumentavam as chances de diagnóstico, ainda que tardiamente. No Caso 2, o rastreamento radiológico apresentou melhor desempenho, inclusive na detecção de suspeitos assintomáticos, como destacam Sanchez et al. 31 e Legrand et al. ${ }^{32}$, mas esbarrou em dificuldades logísticas que impediram a continuidade da ação. Além disso, o exame de contatos era inviável, em virtude do grande número de prisioneiros por cela, aspecto também mencionado por Larouze et al. 33 . A detecção e acompanhamento dos casos por intermédio da microscopia de escarro está implantada, bem como a garantia de medicamentos padronizados.

Ainda que sua efetividade seja objeto de discussão, a estratégia DOTS continua a ser recomendada pela OMS 34 . O primeiro de seus componentes, o compromisso político com a mobilização de recursos para o controle da TB, essencial para a operacionalização das ações, tem-se mostrado deficiente no sistema prisional ${ }^{33}$. O tratamento diretamente observado, implantado nas UA do Caso 1, não é previsto nas do Caso 2 sob o argumento de que pode parecer coercitivo e autoritário para uma população já submetida a várias pressões. Um modelo menos rígido de tratamento diretamente observado, com apoio às necessidades do paciente e incentivo ao seu tratamento pode alcançar melhores resultados 35 .

A percepção dos agentes de segurança sobre os direitos dos encarcerados não difere, em estudo recente, da apresentada pela maior parte da população brasileira, que demonstra hostilidade aos direitos dos apenados e apoia medidas de endurecimento da execução penal 25,36. Os agentes de segurança que haviam recebido treinamento sobre $\mathrm{TB}$ ou eram profissionais da área de saúde reconheciam a importância das ações de saúde para o indivíduo e para a coletividade. Atividades educativas e de sensibilização são necessárias para os agentes de segurança e profissionais administrativos das prisões, para que a tuberculose seja uma preocupação compartilhada 33 .

A análise de implantação permitiu especificar o conjunto dos fatores que facilitam ou comprometem a implantação de uma intervenção 37 e discriminar a contribuição dos componentes verdadeiramente implantados do programa para a produção dos efeitos 15 . Essa avaliação foi útil para compreender o que contribui para os aspectos exitosos do programa, para o alcance de suas metas e para identificar seus limites 38 .
A definição, baseada nas diretrizes para o controle da TB, de critérios e parâmetros explícitos a priori para guiar o julgamento, permitiu uma análise objetiva das categorias escolhidas para avaliar o grau de implantação do programa. A valoração é estabelecida não só sobre o observado, mas valendo-se do que é construído sobre o observado, com base em conhecimento e experiências de outros atores 39,40. A validação dos critérios, dos parâmetros e da pontuação utilizados na matriz de análise e julgamento é possível por meio do consenso entre avaliadores, gestores e técnicos do programa 41,42,43. No entanto, a construção do consenso pelas oficinas para análise dos resultados e definição de indicadores não foi possível neste estudo, devido à dificuldade institucional dos atores em participar de todo o processo. O grupo de pesquisa optou por atribuir o mesmo valor a todas as categorias, por considerá-las igualmente relevantes.

A pequena mobilização dos atores envolvidos na saúde do sistema penitenciário durante este trabalho avaliativo leva-nos a questionar o seu potencial como veículo de mudanças. Os resultados de uma avaliação não geram automaticamente uma decisão, mas espera-se que as informações produzidas possam contribuir para o julgamento de uma intervenção por parte das instâncias de decisão e influir positivamente nas suas intenções estratégicas.

Com base na análise de implantação feita neste estudo, as recomendações em vigor, que demandam maior atenção, podem ser sintetizadas: que as ações de busca ativa sejam enfatizadas tanto no ingresso do detento como por meio de exames periódicos e dos contatos; que o tratamento diretamente observado seja feito para todos de forma a esclarecer dúvidas, promover a educação em saúde e evitar o uso indevido dos medicamentos; e que a comunicação entre os profissionais de segurança e os da saúde nas unidades prisionais, entre as unidades prisionais por ocasião das transferências, e entre o sistema penitenciário e o sistema de saúde nas situações de livramento, seja feita de forma a evitar a interrupção do tratamento dos pacientes, aumentando as taxas de cura e, consequentemente, reduzindo a transmissão da doença no ambiente prisional. Em última análise, o impacto desse conjunto de medidas pode beneficiar a própria equipe de saúde ao propiciar evolução favorável dos casos e reduzir a transmissão da doença.

O estudo demonstrou que o modelo teórico proposto poderá ser utilizado em outros locais (replicação do modelo), visto que a orientação normativa para o controle da tuberculose prisional é comum para todo o país, ainda que haja particularidades. 


\section{Resumen}

En Brasil, los esfuerzos para controlar la tuberculosis en la población carcelaria desde hace diez años se regulan mediante el Plan Nacional de Salud para el sistema penitenciario. Los estados tienen diferentes modelos de organización del Programa de Control de Tuberculosis (PCT) en el sistema penitenciario. En este estudio se evaluó el grado de implantación del PCT en las cárceles de dos estados. Se procedió a un estudio de casos múltiples con un enfoque cualitativo y el desarrollo de una matriz de análisis y juicio. De acuerdo con los criterios predefinidos, se seleccionaron 2 casos estatales, con 2 unidades de análisis para cada caso y 1 hospital penitenciario en el caso 2. La implantación parcial del programa se identificó en las cárceles del caso 1 y el hospital; un bajo nivel de implantación se encontró en las prisiones del caso 2. La falta de inversión financiera y de recursos, de integración entre el ámbito de la justicia y salud, y el escaso acceso a los servicios de salud, fueron algunos de los factores adversos para el establecimiento del programa.

Tuberculosis; Prisiones; Evaluación de Programas y Proyectos de Salud

\section{Referências}

1. World Heatlh Organization. Global tuberculosis report 2013. Geneva: World Heatlh Organization; 2013.

2. Pan American Health Organization. Guía para el control de la tuberculosis en poblaciones privadas de libertad de América Latina y el Caribe. Washington DC: Pan American Health Organization; 2008.

3. Secretaria de Vigilância em Saúde, Ministério da Saúde. PNCT: manual de recomendações para o controle da tuberculose no Brasil. Brasília: Ministério da Saúde; 2010.

4. Barreto ML, Teixeira MG, Bastos FI, Ximenes RAA, Barata RB, Rodrigues LC. Successes and failures in the control of infectious diseases in Brazil: social and environmental context, policies, interventions, and research needs. Lancet 2011. http:// www.thelancet.com/series/health-in-brazil (acessado em 20/Jun/2013).

\section{Colaboradores}

L. G. D. Oliveira participou da concepção do projeto de pesquisa, análise e interpretação dos dados e redação do artigo. S. Natal colaborou na concepção do projeto de pesquisa, redação do artigo e aprovação final da versão a ser publicada. L. A. B. Camacho contribuiu na revisão crítica do conteúdo e aprovação final da versão a ser publicada.

\section{Agradecimentos}

Agradecemos ao Allan Nuno Alves de Sousa pela valiosa participação na coleta de dados. Ao CNPq (edital 67/ $2009 \mathrm{MCT} / \mathrm{CNPq}$ /CT-MS/DECIT), pelo financiamento.
5. Sanchez AR. Tuberculose em população carcerária do Estado do Rio de Janeiro: prevalência e subsídios para formulação de estratégia de controle [Tese de Doutorado]. Rio de Janeiro: Escola Nacional de Saúde Pública Sergio Arouca, Fundação Oswaldo Cruz; 2007.

6. Parvez FM. Prevention and control of tuberculosis in correctional facilities. In: Greifinger RB, editor. Public health behind bars: from prisons to communities. New York: Springer; 2007. p. 174-211.

7. World Health Organization. Tuberculosis in prisons. Geneva: World Health Organization; 2013.

8. Ruiz F, Lopez G. Documento de consenso para el control de la tuberculosis en las prisiones españolas. Rev Esp Sanid Penit 2010; 12:64-78.

9. Departamento de Ações Programáticas Estratégicas, Secretaria de Atenção à Saúde, Ministério da Saúde. Legislação em saúde no sistema penitenciário. Brasília: Ministério da Saúde; 2010. 
10. Oliveira LGD, Natal S, Camacho LAB. O programa de controle da tuberculose em unidades prisionais de dois estados brasileiros. Cad Saúde Colet (Rio J.) 2012; 20:250-7.

11. Yin RK. Estudo de caso: planejamento e métodos. 3a Ed. Porto Alegre: Bookman; 2005.

12. Oliveira LGD, Natal S, Santos EM. Avaliação de implantação do Programa de Controle da Tuberculose no Município de Niterói/RJ. Rev Bras Pneumol Sanit 2007; 15:29-38.

13. Natal S, Hartz Z, Santos EM, Penna ML, Sabroza P. Avaliação do Programa de Controle da Tuberculose: estudo de casos na Amazônia legal. Bol Pneumol Sanit 2004; 12:91-109.

14. Penchansky DBA, Thomas JW. The concept of access: definition and relationship to consumer satisfaction. Med Care 1981; 19:127-40.

15. Bardin L. Análise de conteúdo. São Paulo: Edições 70; 2011.

16. Natal S, Santos EM, Alves CKA, Felisberto E, Santos ES, Sousa A. A implementação do Projeto Estratégico de Fortalecimento da Capacidade Técnica em Monitoramento e Avaliação das Secretarias de Saúde. In: Hartz ZMA, Felisberto E, Vieira da Silva LM, organizadores. Meta-avaliação da atenção básica à saúde. Rio de Janeiro: Editora Fiocruz; 2008. p. 341-67.

17. Denis JL, Champagne F. Análise de implantação. In: Hartz ZMA, organizadora. Avaliação em saúde: dos modelos conceituais à prática na análise da implantação de programas. Rio de Janeiro: Editora Fiocruz; 1997. p. 49-88.

18. Reichard AA, Lobato MN, Roberts CA, Bazerman LB, Hammett TM. Assessment of tuberculosis screening and management practices of large jail systems. Public Health Rep 2003; 118:500-7.

19. Balabanova Y, Drobniewski F, Fedorin I, Zakharova S, Nikolayevskyy V, Atun R, et al. The Directly Observed Therapy Short-Course (DOTS) strategy in Samara Oblast, Russian Federation. Respir Res 2006; 7:44.

20. Aerts A, Hauer B, Wanlin M, Veen J. Tuberculosis and tuberculosis control in European prisons. Int J Tuberc Lung Dis 2006; 10:1215-23.

21. Sosa LE, Lobato MN, Condren T, Williams MN, Hadler JL. Outbreak of tuberculosis in a correctional facility: consequences of missed opportunities. Int J Tuberc Lung Dis 2008; 12:689-91.

22. Harries AD, Nyirenda TE, Yadidi AE, Gondwe MK, Kwanjana JH, Salaniponi FM. Tuberculosis control in Malawian prisons: from research to policy and practice. Int J Tuberc Lung Dis 2004; 8:614-17.

23. Kanyerere HS, Banda RP, Gausi F, Salaniponi FM, Harries AD, Mpunga J, et al. Surveillance of tuberculosis in Malawian prisons. Public Health Action 2012; 2:10-4

24. Souza KMJ, Villa TCS, Assolini FEP, Sá LD, Teixeira PF, Palha PF. Atraso no diagnóstico da tuberculose: sistema prisional. In: Villa TCS, Ruffino Netto A, organizadores. Diagnóstico da tuberculose: avaliação dos serviços de saúde em municípios de diferentes regiões do Brasil. Ribeirão Preto: FUNPEC Editora; 2011. p. 139-44.
25. Magalhães CA, Moura E. Direitos humanos, pena de morte e sistema prisional. In: Venturi G, organizador. Direitos humanos: percepções da opinião pública. Brasília: Secretaria de Direitos Humanos; 2010. p. 76-91.

26. Castro VD. Saúde nas prisões: um estudo da implementação do programa de controle da tuberculose em uma unidade do sistema penitenciário [Dissertação de Mestrado]. Rio de Janeiro: Escola Nacional de Saúde Pública Sergio Arouca, Fundação Oswaldo Cruz; 2011.

27. Kranzer K, Afnan-Holmes H, Tomlin K, Golub JE, Shapiro AE, Schaap A, et al. The benefits to communities and individuals of screening for active tuberculosis disease: a systematic review. Int J Tuberc Lung Dis 2013; 17:432-46.

28. Golub JE, Dowdy DW. Screening for active tuberculosis: methodological challenges in implementation and evaluation. Int J Tuberc Lung Dis 2013; 17:856-65.

29. Lönnroth K, Corbett E, Golub J, Godfrey-Faussett P, Uplekar M, Weil D, et al. Systematic screening for active tuberculosis: rationale, definitions and key considerations. Int J Tuberc Lung Dis 2013; 17:28998.

30. Schneeberger Geisler S, Helbling P, Zellweger JP, Altpeter ES. Screening for tuberculosis in asylum seekers: comparison of chest radiography with an interview-based system. Int J Tuberc Lung Dis 2010; 14:1388-94.

31. Sanchez A, Gerhardt G, Natal S, Capone D, Espinola A, Costa W, et al. Prevalence of pulmonary tuberculosis and comparative evaluation of screening strategies in a Brazilian prison. Int J Tuberc Lung Dis 2005; 9:633-9.

32. Legrand J, Sanchez A, Le Pont F, Camacho L, Larouze B. Modeling the impact of tuberculosis control strategies in highly endemic overcrowded prisons. PLoS One 2008; 3:e2100.

33. Larouze B, Sanchez A, Diuana V. Tuberculosis behind bars in developing countries: a hidden shame to public health. Trans R Soc Trop Med Hyg 2008; 102:841-2.

34. World Health Organization. Treatment of tuberculosis: guidelines. $4^{\text {th }}$ Ed. Geneva: World Health Organization; 2010.

35. Oliveira LGD, Natal S, Chrispim PPM. Tratamento diretamente supervisionado: estratégia para o controle da tuberculose. Rev APS 2010; 13:357-64.

36. Cano I. Direitos humanos, criminalidade e segurança pública. In: Venturi G, organizador. Direitos humanos: percepções da opinião pública. Brasília: Secretaria de Direitos Humanos; 2010. p. 65-75.

37. Champagne F, Brousselle A, Hartz Z, Contandriopoulos A-P, Denis JL. A análise da implantação. In: Brousselle A, Champagne F, Contandriopoulos A-P, Hartz Z, organizadores. Avaliação: conceitos e métodos. Rio de Janeiro: Editora Fiocruz; 2011. p. 21738.

38. Minayo MCS. Conceito de avaliação por triangulação de métodos. In: Minayo MCS, Assis SG, Souza ER, organizadores. Avaliação por triangulação de métodos: abordagem de programas sociais. Rio de Janeiro: Editora Fiocruz; 2005. p. 19-51. 
39. Santos SEM, Cruz MM, Decotelli PV, Chrispim PPM, Abreu DMF. Avaliadores in situ (ação). Reflexões e desafios sobre a institucionalização da prática avaliativa no PN DST/Aids. In: Campos RO, Furtado JP, organizadores. Desafios da avaliação de programas e serviços em saúde: novas tendências e questões emergentes. Campinas: Editora da Unicamp; 2011. p. 205-35.

40. Ristoff DI. Algumas definições de avaliação. Revista da Rede de Avaliação Institucional de Educação Superior 2003; 8:19-30.

41. Assis SG, Deslandes SF, Minayo MCS, Santos NC. Definição de objetivos e construção de indicadores visando à triangulação. In: Minayo MCS, Assis SG, Souza EC, organizadores. Avaliação por triangulação de métodos: abordagem de programas sociais. Rio de Janeiro: Editora Fiocruz; 2005. p. 105-32.
42. Medina MG, Silva GAP, Aquino R, Hartz ZMA. Uso de modelos teóricos na avaliação em saúde: aspectos conceituais e operacionais. In: Hartz ZMA, Vieira da Silva LM, organizadoras. Avaliação em saúde: dos modelos teóricos à prática na avaliação de programas e sistemas de saúde. Salvador: EDUFBA/Rio de Janeiro: Editora Fiocruz; 2005. p. 41-63.

43. Alves CKA, Assis SG, Souza ER. Interpretação e análise das informações: o uso de matrizes, critérios, indicadores e padrões. In: Samico I, Felisberto E, Figueiró AC, Frias PG, organizadores. Avaliação em saúde: bases conceituais e operacionais. Rio de Janeiro: Medbook Editora; 2010. p. 89-107.

Recebido em 18/Mar/2014

Versão final reapresentada em 16/Set/2014

Aprovado em 29/Set/2014 